\title{
Light Quark Mass Dependence in Heavy Quarkonium Physics
}

\author{
Feng-Kun Guo ${ }^{1, *}$ and Ulf-G. Meißner ${ }^{1,2, \dagger}$ \\ ${ }^{1}$ Helmholtz-Institut für Strahlen- und Kernphysik and Bethe Center for Theoretical Physics, Universität Bonn, \\ D-53115 Bonn, Germany \\ ${ }^{2}$ Institute for Advanced Simulation, Institut für Kernphysik and Jülich Center for Hadron Physics,
}

Forschungszentrum Jülich, D-52425 Jülich, Germany

(Received 10 March 2012; published 10 August 2012)

\begin{abstract}
The issue of chiral extrapolations in heavy quarkonium systems is discussed. We show that the light quark mass dependence of the properties of heavy quarkonia is not always suppressed. For quarkonia close to an open flavor threshold, even a nonanalytic chiral extrapolation is needed. Both these nontrivial facts are demonstrated to appear in the decay widths of the hindered $M 1$ transitions between the first radially excited and ground state $P$-wave charmonia. The results at a pion mass of about $500 \mathrm{MeV}$ could deviate from the value at the physical pion mass by a factor of two. Our findings show the necessity of performing chiral extrapolations for lattice simulations of heavy quarkonium systems. Furthermore, lattice calculations of these transitions would also provide a definite answer to the role of coupled-channel effects in heavy quarkonium physics due to virtual heavy mesons.
\end{abstract}

DOI: 10.1103/PhysRevLett.109.062001

PACS numbers: 14.40.Pq, 12.38.Gc, 13.25.Gv

Since the discovery of $J / \psi$, the physics of heavy quarkonium is an important tool for testing QCD. Because both the charm and bottom quark masses are much larger than the nonperturbative scale $\Lambda_{\mathrm{QCD}}$, heavy quarkonia were well described in the framework of potential models. However, in recent years this simple picture has been shattered, as quite a few charmonium states close to or above the open charm thresholds were discovered, and many of their properties are not expected from the potential models. For a recent comprehensive review, see Ref. [1]. The spectrum of heavy quarkonium has been intensively studied using lattice simulations, using the quenched approximation in the early stages, and in full QCD in recent years; for example, see Refs. [2-9]. While most of the calculations focus on the low-lying states, which we refer to as the states below open heavy flavor thresholds, only a few calculations tackle the problem of higher excited states [8-10]. So far, all calculations of heavy quarkonium in full QCD are performed at light quark masses larger than the physical values, or equivalently with unphysical pion masses. The simulations in Ref. [9] are only performed at a single pion mass $M_{\pi}=396 \mathrm{MeV}$. Mixing of charmonia with pairs of open charm states are taken into account in Ref. [8] at three different pion masses ranging from $1 \mathrm{GeV}$ down to $280 \mathrm{MeV}$, yet no chiral extrapolation to the physical pion mass was performed. In addition to the spectrum, there have also been lattice simulations of the charmonium $[11,12]$ and bottomonium radiative transitions [13]. The quenched approximation is used in Ref. [11], and the calculations of Ref. [12] were performed at $M_{\pi}=485 \mathrm{MeV}$.

Being bound states of a heavy quark and heavy antiquark, heavy quarkonia do not contain any valence light quark. Thus, one would naively expect that the light quark mass dependence of their properties would be suppressed, so that one can use a simple linear formula in the light quark masses [remember for example that $M_{\pi}^{2} \propto\left(m_{u}+m_{d}\right)$ at leading order] for chiral extrapolation, as in Refs. [4,5] for mass splittings. While this is true for low-lying states, a similar simple extrapolation may not be reasonable for higher, excited states. The purpose of this Letter is to show that dramatic and even nonanalytic dependences in the light quark masses can arise. Hence, for the excited states that are close to open flavor thresholds, a formula that takes into account the nonanalyticity should be utilized for chiral extrapolation. Furthermore, for radiative transitions with strong coupled-channel effects, simulations at several pion masses are necessary to extract the physical results.

The effects of light quarks in heavy quarkonium systems are due to quantum fluctuations of the sea quarks. Sea quark and antiquark pairs are created and annihilated in the color singlet heavy quarkonium. Low-energy fluctuations can be described in the framework of chiral perturbation theory, which is the standard tool for chiral extrapolations. The quarkonium states can be included as matter fields. Let us focus on the quark mass dependence of the quarkonium mass. Two types of sea quark fluctuations are schematically depicted in Fig. 1; type (a) is disconnected and suppressed according to the Okuba-ZweigIizuka rule; type (b) means that the heavy quark (antiquark)

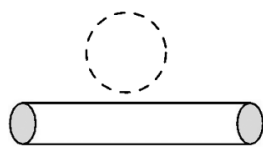

(a)

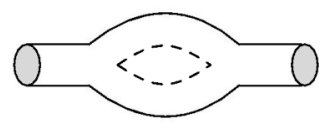

(b)
FIG. 1. Schematic diagrams of the creation and annihilation of sea quarks in a heavy quarkonium, with solid and dashed lines representing heavy quarks and light sea quarks, respectively. 
and virtual sea antiquark (quark) can form a color singlet state, a heavy meson (antimeson); i.e., a virtual-heavymeson-antimeson pair is created and annihilated after a short propagation. There are certainly other contributions, such as the doubly Okuba-Zweig-Iizuka suppressed processes that induce mixing of the heavy quarkonium and a light meson. We expect that such contributions are less important, and therefore do not consider them. Type (a) can be parameterized using an effective chiral Lagrangian containing unknown low-energy constants. The resulting quark mass dependence is analytic in the light quark masses up to chiral logarithms, see Ref. [14]. For instance, denoting the operator annihilating a quarkonium field by $\psi$, a possible contribution would be proportional to $\psi^{\dagger} \psi\left\langle\chi_{+}\right\rangle$in the effective chiral Lagrangian. Here, $\chi_{+}=$ $u^{\dagger} \chi u^{\dagger}+u \chi u,\langle\rangle$ is the flavor trace, and $\chi=2 B$ diag $\left(m_{u}, m_{d}, m_{s}\right)$ contains the light quark mass matrix, where $B=|\langle 0|\bar{q} q| 0\rangle| / F^{2}$ and $F$ is the pion decay constant in the chiral limit. The Goldstone boson field $\Phi$, which contains the pions, eta and kaons in the SU(3) case, are included in $u=\sqrt{U}$ and $U=\exp (i \sqrt{2} \Phi / F)$. At leading order $\mathcal{O}\left(M_{\pi}^{2}\right)$, this term gives a contribution proportional to $B\left(m_{u}+\right.$ $\left.m_{d}+m_{s}\right)$ to the quarkonium mass, and at $\mathcal{O}\left(M_{\pi}^{4}\right)$, the chiral $\log$ arithm $M_{\pi}^{4} \log M_{\pi}^{2}$ will arise.

Complexity comes from type (b), which can lead to nonanalyticity, as will be shown below. Because the heavy quarkonium states are normally not far from the open flavor thresholds, the open flavor mesons, at least the ground states, do not necessarily decouple in low-energy effective field theory (EFT) for heavy quarkonium. In particular, the masses of many excited quarkonium states are very close to the thresholds. In that case, one should consider coupled-channel effects due to coupling to the open flavor mesons and antimesons in chiral extrapolation. As an example, let us study $P$-wave charmonium states. They couple to the pseudoscalar and vector charmed mesons in an $S$-wave with a coupling constant $g$. The selfenergy due to coupling to the charmed mesons with masses $m_{1}$ and $m_{2}$ is expressed in terms of the scalar two-point loop function

$$
\Sigma\left(P^{2}\right) \equiv \int \frac{d^{4} l}{(2 \pi)^{4}} \frac{i}{\left(l^{2}-m_{1}^{2}+i \epsilon\right)\left[(P-l)^{2}-m_{2}^{2}+i \epsilon\right]} .
$$

In the rest frame of the charmonium and taking the nonrelativistic approximation for both propagators, we regularize the divergent loop with a three-momentum cutoff $\lambda$

$$
\Sigma\left(M^{2}, \lambda\right)=\frac{1}{4 \pi\left(m_{1}+m_{2}\right)}\left(-\frac{\lambda}{\pi}+\frac{1}{2} \sqrt{c-i \epsilon}\right),
$$

where $M$ denotes the charmonium mass, $c=2 \mu_{12} b_{12}$ with $\mu_{i j}=m_{i} m_{j} /\left(m_{i}+m_{j}\right)$ the reduced mass, and $b_{12}=$ $m_{1}+m_{2}-M$. The mass gets renormalized by the real part of the self-energy. Writing out the $M_{\pi}$-dependence explicitly, we get

$$
M\left(M_{\pi}\right)=M_{0}\left(\lambda, M_{\pi}\right)+g^{2} m_{1} m_{2} \operatorname{Re} \Sigma\left(M^{2}, \lambda, M_{\pi}\right),
$$

where the factor $m_{1} m_{2}$ is required for correct normalization, and

$$
M_{0}\left(\lambda, M_{\pi}\right)=\stackrel{\circ}{M}_{0}(\lambda)+d(\lambda) M_{\pi}^{2}+\mathcal{O}\left(M_{\pi}^{4}\right)
$$

is the bare mass. (Note that the mass shift due to virtual loops is a scale-dependent quantity, as can be seen in Eq. (3), and not a physical observable. For phenomenological studies of the charmed meson loops in the charmonium spectrum, we refer to Refs. [15,16].) Both the chiral-limit bare mass $\stackrel{\circ}{M}_{0}(\lambda)$ and the coefficient $d(\lambda)$ depend on the cutoff, since the masses of open flavor heavy mesons $m_{1}$ and $m_{2}$ depend on the pion mass. For simplicity, we assume that the heavy mesons lie in the same spin multiplet. Up to $\mathcal{O}\left(M_{\pi}^{2}\right)$, we have $m_{i}=\stackrel{\circ}{m}_{i}+h_{1} M_{\pi}^{2} / \stackrel{\circ}{m}_{i}$ [17], where $h_{1}$ is a dimensionless coefficient of order unity. Notice that

$$
\sqrt{c}=\sqrt{2 \stackrel{\circ}{\mu}_{12}\left(\delta+\frac{h_{1}}{\stackrel{\circ}{\mu}_{12}} M_{\pi}^{2}\right)+\mathcal{O}\left(M_{\pi}^{4}\right)},
$$

where $\stackrel{\circ}{\mu}_{12}=\stackrel{\circ}{m}_{1} \stackrel{\circ}{m}_{2} /\left(\stackrel{\circ}{m}_{1}+\stackrel{\circ}{m}_{2}\right)$, and $\delta=\stackrel{\circ}{m}_{1}+\stackrel{\circ}{m}_{2}-M$. Therefore, for the case with $|\delta| \lesssim R \equiv M_{\pi}^{2} / \stackrel{\circ}{\mu}_{12}$, the unitary cut in the loop function Eq. (2) cannot be expanded in a polynomial in $M_{\pi}$. Although $R \simeq 20 \mathrm{MeV}$ is small at the physical pion mass, it is around $270 \mathrm{MeV}$ for a pion mass of $500 \mathrm{MeV}$. As a result, there will be a cusp due to the nonanalyticity at the point $M\left(M_{\pi}\right)=m_{1}\left(M_{\pi}\right)+m_{2}\left(M_{\pi}\right)$. Nonanalyticity due to similar effects in the chiral extrapolation was discussed earlier for the $\Delta$ resonance $[18,19]$ and the pion form factor [20].

We use $h_{1}=0.44$ as determined from the $\mathrm{SU}(3)$ mass splittings of both the pseudoscalar and vector charmed mesons $[21,22]$. The $M_{\pi}$-dependence of $M-M_{0}(\lambda)$ for the first radially excited $P$-wave charmonia is plotted in Fig. 2 (upper), where $\lambda=0.63 \mathrm{GeV}$, corresponding to $M-M_{0}(\lambda)=0$ for the $h_{c}^{\prime}$ at the physical pion mass, is used; $g_{1}^{\prime}$ is the coupling of the $2 P$ charmonia to the charmed mesons, as defined in [23], and its dimension is mass $^{-1 / 2}$. Using model values for the masses of $\chi_{c 0}^{\prime}, \chi_{c 1}^{\prime}$, and $h_{c}^{\prime}$ at the physical pion mass from Ref. [24], these states are above the coupled thresholds at the physical pion mass. Increasing the pion mass, the charmed meson masses increase, too. One expects the charmonium mass to increase more slowly than the charmed meson thresholds. Therefore, for a charmonium with a mass slightly higher than the open charm threshold at the physical pion mass, the charmonium mass should coincide with the threshold at some larger pion mass. After that, the open charm mesons cannot go on shell, and a cusp shows up because of the end of the unitary cut, as seen in Fig. 2 (upper); $\chi_{c 2}^{\prime}$ is always below the $D^{*} \bar{D}^{*}$ threshold, so that there is no cusp in the curve for this state. 

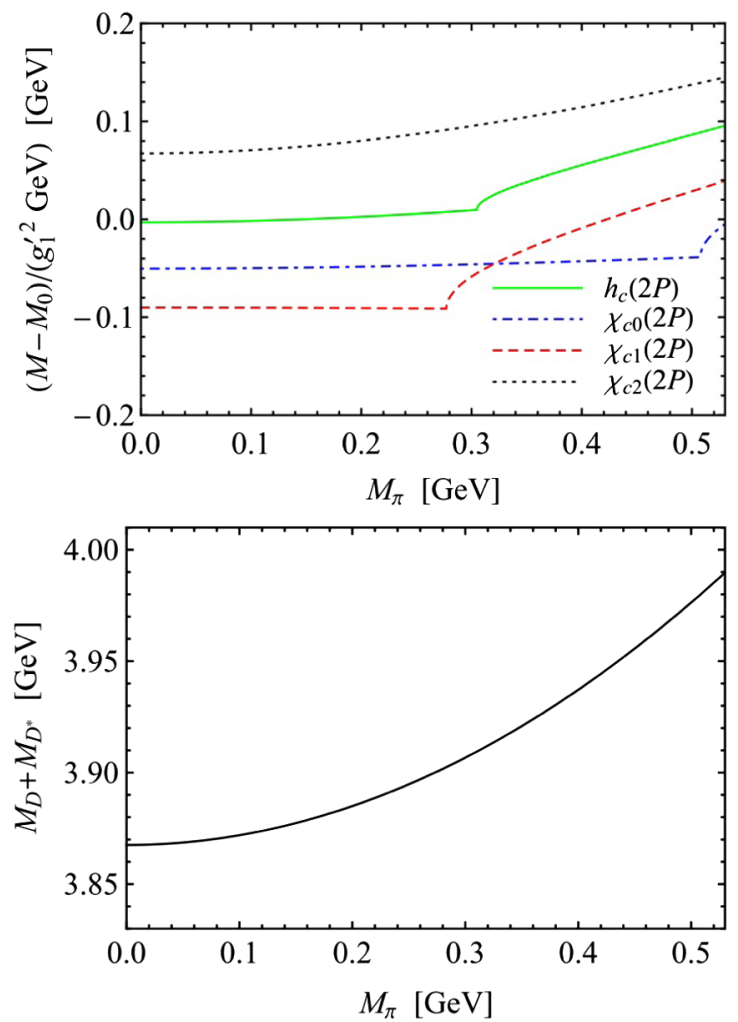

FIG. 2 (color online). Upper panel: Pion mass dependence of $M-M_{0}(\lambda)$ for the $2 P$ charmonia calculated with $\lambda=0.63 \mathrm{GeV}$. Lower panel: Pion mass dependence of the $D \bar{D}^{*}$ threshold.

For an $S$-wave charmonium, the nonanalyticity due to coupling to the pseudoscalar and vector charmed mesons is less important, and even invisible. This is because the coupling is in a $P$-wave. Of course, it can couple to a ground state charmed meson and an orbitally excited state in an $S$-wave, as considered in Ref. [8]. However, the thresholds are far from the masses of the $1 S$ and $2 S$ charmonia. In this case, the square root in Eq. (5) can be expanded in a polynomial in $M_{\pi}^{2}$. Hence, the $M_{\pi}$-dependence of the quarkonium mass is given by Eq. (4) with redefined $\stackrel{\circ}{M}_{0}(\lambda)$ and $d(\lambda)$.

It is instructive to briefly discuss possible hadronic molecules with a binding energy much smaller than the pion mass. In this case, the bound state can be described by an EFT with only contact terms analogous to that for the deuteron; for example, see [25]. Then, the pion mass dependence of the mass of the hadronic molecule is dominated by that of the masses of the constituents, as argued in Ref. [26]. So, in the pion mass range where $X$ (3872) is a $D \bar{D}^{*}$ bound state [27], the $M_{\pi}$-dependence of its mass should be approximately given by that of the threshold, as depicted in Fig. 2 (lower) at $\mathcal{O}\left(M_{\pi}^{2}\right)$. We will not calculate the deviation from the threshold due to a small but finite binding energy here, but only point out that a loosely bound state can easily become unbound by varying

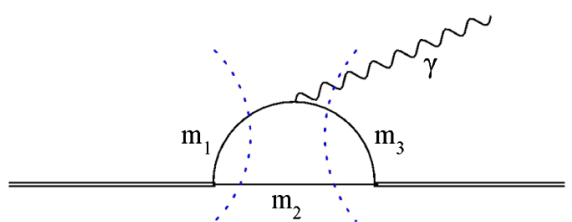

FIG. 3 (color online). Hadronic loop diagram, with the double, solid, and wiggly lines denoting the charmonia, charmed mesons, and photon, respectively. The dashed curves represent the unitary cuts.

the interaction strength. It is worth notice that the coupling constant $g$ in Eq. (3), which controls the strength of the cusp in the $M_{\pi}$-dependence of the charmonium mass, is also a measure of the hadronic molecular content of a given state $[28,29]$. However, it is obvious that a quantitative treatment of the quark mass dependence of a hadronic molecule requires a more refined approach than that given here. For example, see Refs. [30,31].

The chiral corrections to the heavy quarkonium mass are always small compared to the mass in the chiral limit. More noticeable is that there exist quantities in heavy quarkonium physics whose pion mass dependence is strong. For these quantities, chiral extrapolation is mandatory. One can imagine that in the mass splittings between two heavy quarkonium states, the great bulk of the chirallimit masses cancels, and the chiral corrections are potentially large. However, as seen in Eq. (4), it is not possible to give a parameter-free prediction for its pion mass dependence even at $\mathcal{O}\left(M_{\pi}^{2}\right)$, since $d(\lambda)$ is scale dependent. A prediction can only be made after fitting the parameters to sufficiently large data. But there are indeed quantities whose quark mass dependence is strong and can be predicted parameter free. A good example is given by the decay widths of the hindered $M 1$ transitions between the $2 P$ and $1 P$ charmonium states. These transitions are shown to be dominated by coupled-channel effects [32] based on a nonrelativistic effective field theory (NREFT) [23,33,34].

As shown in Ref. [32], these transitions are dominated by triangle diagrams at the hadronic level, with three intermediate charmed or anticharmed mesons (see Fig. 3). The decay amplitude is proportional to the threepoint scalar loop function. It is convergent, and the nonrelativistic expression reads as $[23,35]$

$$
\begin{aligned}
I(q) \equiv & i \int \frac{d^{4} l}{(2 \pi)^{4}} \frac{1}{\left(l^{2}-m_{1}^{2}+i \epsilon\right)\left[(P-l)^{2}-m_{2}^{2}+i \epsilon\right]} \\
& \times \frac{1}{\left[(l-q)^{2}-m_{3}^{2}+i \epsilon\right]} \\
= & \mathcal{N} \frac{1}{\sqrt{a}}\left[\arctan \left(\frac{c^{\prime}-c}{2 \sqrt{a(c-i \epsilon)}}\right)\right. \\
& \left.+\arctan \left(\frac{2 a+c-c^{\prime}}{2 \sqrt{a\left(c^{\prime}-a-i \epsilon\right)}}\right)\right],
\end{aligned}
$$


where $P$ and $q$ are the momenta of the initial particle and the photon, respectively, $b_{23}=m_{2}+m_{3}+q^{0}-M$ with $M$ the mass of the initial particle, $\mathcal{N}=\mu_{12} \mu_{23} /$ $\left(16 \pi m_{1} m_{2} m_{3}\right)$,

$$
a=\left(\frac{\mu_{23}}{m_{3}}\right)^{2} \vec{q}^{2}, \quad c^{\prime}=2 \mu_{23} b_{23}+\frac{\mu_{23}}{m_{3}} \vec{q}^{2},
$$

and $c$ is as defined below Eq. (2). For small $a$, one may expand the loop function out

$I(q)=\mathcal{N} \frac{2}{\sqrt{c^{\prime}}+\sqrt{c}}\left[1+\mathcal{O}\left(\frac{a}{\min \left(c^{\prime},\left(c^{\prime}-c\right) / 2\right)}\right)\right]$.

It is then clear that two unitary cuts (see Fig. 3) lead to the main contribution of the three-point loop. Corresponding to the two cuts, one may define two velocities of the intermediate mesons. The velocity $v$ used in the NREFT power counting [23] should be understood as the average of these two velocities. Following the discussion around Eq. (5), we can expect cusps in the $M_{\pi}$-dependence of the decay widths when the mass of the decaying particle coincides with the coupled threshold. The results for $\Gamma\left(\chi_{c 2}^{\prime} \rightarrow h_{c} \gamma\right)$ and $\Gamma\left(h_{c}^{\prime} \rightarrow \chi_{c J} \gamma\right)$ are shown in Fig. 4, where we have neglected the $M_{\pi}$-dependence of the charmonium masses and used the same model value $3908 \mathrm{MeV}$ for $M_{h_{c}^{\prime}}[24]$ as before.

The cusp in $\Gamma\left(h_{c}^{\prime} \rightarrow \chi_{c J} \gamma\right)$ appears at about $M_{\pi}=$ $300 \mathrm{MeV}$, the same value as in Fig. 2 for $h_{c}^{\prime}$. From Fig. 4, one finds a strong dependence on the pion mass. The value of $\Gamma\left(h_{c}^{\prime} \rightarrow \chi_{c 0} \gamma\right)$ at a pion mass of $485 \mathrm{MeV}$, the same value as that used in the lattice simulations for the $M 1$ transitions between $S$-wave charmonia [12], is only about half of that at the physical pion mass. This observation highlights the necessity of chiral extrapolation of lattice simulations for radiative transitions of heavy quarkonia and the necessity of small pion masses in the simulations. Otherwise, the uncertainty due to unphysical pion mass could be very large. Although parameter-free predictions for $M 1$ transitions between the $S$-wave heavy quarkonia

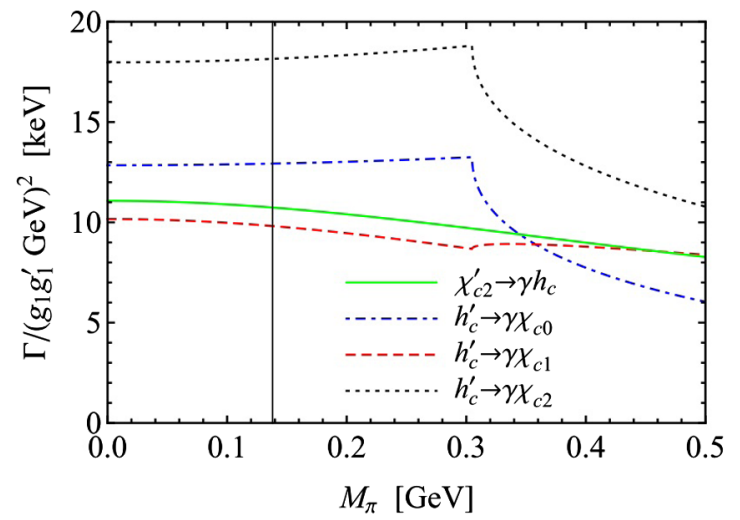

FIG. 4 (color online). Dependence of the widths of various hindered $M 1$ transitions on the pion mass. The vertical line denotes the physical pion mass. are not possible, as noted in Ref. [32], the charmed meson loops are still expected to be crucial [36,37]. Thus, the pion mass dependence induced by the virtual charmed mesons could introduce a large uncertainty due to the large pion mass used in lattice simulations.

One can further study the strange quark mass dependence, which translates into the dependence on $\hat{M}_{K}^{2}=$ $B m_{s}$, where we use the same notation as Ref. [38]. As an example, we plot the simultaneous dependence on $M_{\pi}$ and $\hat{M}_{K}$ of $\Gamma\left(\chi_{c 2}^{\prime} \rightarrow \gamma h_{c}\right)$ and $\Gamma\left(h_{c}^{\prime} \rightarrow \gamma \chi_{c 0}\right)$ in Fig. 5, where we used $M_{D_{s}^{(*)}}=\stackrel{\circ}{M}_{D^{(*)}}+2 h_{1} \hat{M}_{K}^{2} / \stackrel{\circ}{M}_{D^{(*)}}+\mathcal{O}\left(\hat{M}_{K}^{4}\right)$. One clearly sees the nonanalyticity in both the $M_{\pi}$ and $\hat{M}_{K}$ dependence of the latter.

In conclusion, we have discussed chiral extrapolations in heavy quarkonium physics, especially for the higher excited states. These states are close to open flavor thresholds. As a result, chiral extrapolation may be nonanalytic. This observation is true for any excited hadron whose mass is in the neighborhood of an $S$-wave-coupled hadronic threshold. For such a state, we propose to perform the chiral extrapolation of the mass using

$$
M\left(M_{\pi}\right)=\stackrel{\circ}{M}+d M_{\pi}^{2}+\sqrt{e+f M_{\pi}^{2}},
$$

where $\stackrel{\circ}{M}, d, e$, and $f$ are parameters to be fit to the lattice data. For states far away from any open flavor threshold, $e$ will be much larger than $f M_{\pi}^{2}$ so that the square root can be expanded, and one may use only the first two terms in the above equation up to $\mathcal{O}\left(M_{\pi}^{2}\right)$. Furthermore, we find that light quark mass dependence is not always suppressed for heavy quarkonium systems. As an example, we show that lattice results for the decay widths of the hindered $M 1$ transitions between $P$-wave charmonia at a pion mass around $500 \mathrm{MeV}$ can deviate by a factor of 2 from the actual values at the physical pion mass. Simulations of these transitions would also provide a nice test of the NREFT, and would be very useful in identifying the coupled-channel effects, which might be the key to understanding some long-standing puzzles in heavy quarkonium systems. If the resulting pion mass dependences follow our predictions, they would also allow for extraction of the product of coupling constants $g_{1} g_{1}^{\prime}$, which cannot be measured directly.
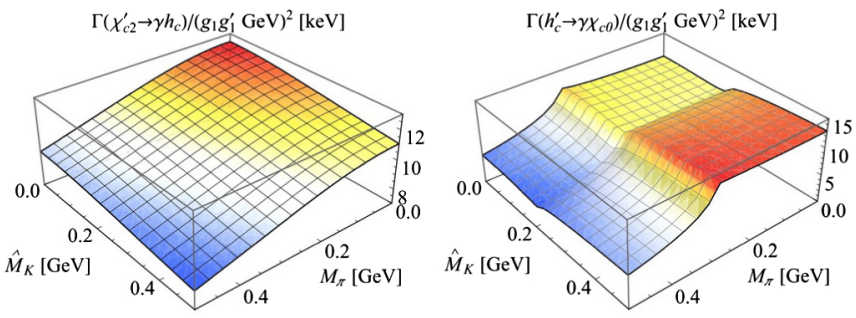

FIG. 5 (color online). $M_{\pi}$ and $\hat{M}_{K}$ dependence of $\Gamma\left(\chi_{c 2}^{\prime} \rightarrow \gamma h_{c}\right)$ (left) and $\Gamma\left(h_{c}^{\prime} \rightarrow \gamma \chi_{c 0}\right)$ (right). 
We would like to thank Hans-Werner Hammer and Christoph Hanhart for valuable discussions. This work is supported in part by the DFG and the NSFC through funds provided to the Sino-German CRC 110 "Symmetries and the Emergence of Structure in QCD" and the EU I3HP "Study of Strongly Interacting Matter" under the Seventh Framework Program of the EU. U.-G. M. Also thanks the BMBF for support (Grant No. 06BN9006). F.-K. G. acknowledges partial support from the NSFC (Grant No. 11165005).

*fkguo@hiskp.uni-bonn.de

†meissner@hiskp.uni-bonn.de

[1] N. Brambilla et al., Eur. Phys. J. C 71, 1534 (2011).

[2] A. Gray, I. Allison, C. T. H. Davies, E. Gulez, G. P. Lepage, J. Shigemitsu, and M. Wingate (HPQCD and UKQCD Collaborations), Phys. Rev. D 72, 094507 (2005).

[3] R. J. Dowdall et al. (HPQCD Collaboration), Phys. Rev. D 85, 054509 (2012).

[4] T. Burch, C. DeTar, M. D. Pierro, A. X. El-Khadra, E. D. Freeland, S. Gottlieb, A. S. Kronfeld, L. Levkova, P. B. Mackenzie, and J. N. Simone (Fermilab Lattice and MILC Collaborations), Phys. Rev. D 81, 034508 (2010).

[5] S. Meinel, Phys. Rev. D 82, 114502 (2010).

[6] D. Mohler and R. M. Woloshyn, Phys. Rev. D 84, 054505 (2011).

[7] J. O. Daldrop, C. T.H. Davies, and R. J. Dowdall, Phys. Rev. Lett. 108, 102003 (2012).

[8] G. S. Bali, S. Collins, and C. Ehmann, Phys. Rev. D 84, 094506 (2011).

[9] L. Liu, G. Moir, M. Peardon, S. Ryan, C. Thomas, P. Vilaseca, J. Dudek, R. Edwards, B. Joo, and D. Richards (Hadron Spectrum Collaboration), arXiv:1204.5425 .

[10] J. J. Dudek, R. G. Edwards, N. Mathur, and D. G. Richards, Phys. Rev. D 77, 034501 (2008).

[11] J. J. Dudek, R. G. Edwards, and C. E. Thomas, Phys. Rev. D 79, 094504 (2009).

[12] Y. Chen et al., Phys. Rev. D 84, 034503 (2011).

[13] R. Lewis and R. M. Woloshyn, Phys. Rev. D 84, 094501 (2011).

[14] B. Grinstein and I. Z. Rothstein, Phys. Lett. B 385, 265 (1996).
[15] E. J. Eichten, K. Lane, and C. Quigg, Phys. Rev. D 73, 014014 (2006).

[16] M. R. Pennington and D. J. Wilson, Phys. Rev. D 76, 077502 (2007); T. Barnes and E. S. Swanson, Phys. Rev. C 77, 055206 (2008); B.-Q. Li, C. Meng, and K.-T. Chao, Phys. Rev. D 80, 014012 (2009).

[17] F. K. Guo, C. Hanhart, and U.-G. Meißner, Eur. Phys. J. A 40, 171 (2009).

[18] V. Bernard, D. Hoja, U.-G. Meißner, and A. Rusetsky, J. High Energy Phys. 06 (2009) 061.

[19] T. Ledwig, V. Pascalutsa, and M. Vanderhaeghen, Phys. Rev. D 82, 091301 (2010).

[20] F. K. Guo, C. Hanhart, F. J. Llanes-Estrada, and U.-G. Meißner, Phys. Lett. B 703, 510 (2011).

[21] F. K. Guo, C. Hanhart, S. Krewald, and U.-G. Meißner, Phys. Lett. B 666, 251 (2008).

[22] J. Hofmann and M.F. M. Lutz, Nucl. Phys. A733, 142 (2004).

[23] F. K. Guo, C. Hanhart, G. Li, U.-G. Meißner, and Q. Zhao, Phys. Rev. D 83, 034013 (2011).

[24] B. Q. Li and K. T. Chao, Phys. Rev. D 79, 094004 (2009).

[25] H.-W. Hammer and L. Platter, Annu. Rev. Nucl. Part. Sci. 60, 207 (2010).

[26] M. Cleven, F. K. Guo, C. Hanhart, and U.-G. Meißner, Eur. Phys. J. A 47, 19 (2011).

[27] N. A. Törnqvist, Phys. Lett. B 590, 209 (2004).

[28] S. Weinberg, Phys. Rev. 130, 776 (1963); 131, 440 (1963); 137, B672 (1965).

[29] V. Baru, J. Haidenbauer, C. Hanhart, Yu. Kalashnikova, and A. E. Kudryavtsev, Phys. Lett. B 586, 53 (2004).

[30] M. T. AlFiky, F. Gabbiani, and A. A. Petrov, Phys. Lett. B 640, 238 (2006).

[31] S. Fleming, M. Kusunoki, T. Mehen, and U. van Kolck, Phys. Rev. D 76, 034006 (2007).

[32] F. K. Guo and U.-G. Meißner, Phys. Rev. Lett. 108, 112002 (2012).

[33] F. K. Guo, C. Hanhart, and U.-G. Meißner, Phys. Rev. Lett. 103, 082003 (2009); 104, 109901(E) (2010).

[34] F. K. Guo, C. Hanhart, G. Li, U.-G. Meißner, and Q. Zhao, Phys. Rev. D 82, 034025 (2010).

[35] M. Cleven, F. K. Guo, C. Hanhart, and U.-G. Meißner, Eur. Phys. J. A 47, 120 (2011).

[36] G. Li and Q. Zhao, Phys. Lett. B 670, 55 (2008); Phys. Rev. D 84, 074005 (2011).

[37] T. Mehen and D.-L. Yang, Phys. Rev. D 85, 014002 (2012).

[38] M. Frink and U.-G. Meißner, J. High Energy Phys. 07 (2004) 028. 